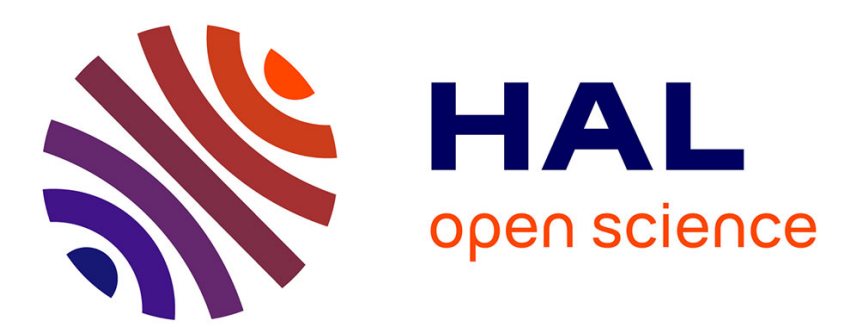

\title{
Effects of particles size on the overall strength of nanocomposites: Molecular Dynamics simulations and theoretical modeling
}

\author{
Antoine Lucchetta, Stella Brach, Djimédo Kondo
}

\section{- To cite this version:}

Antoine Lucchetta, Stella Brach, Djimédo Kondo. Effects of particles size on the overall strength of nanocomposites: Molecular Dynamics simulations and theoretical modeling. Mechanics Research Communications, 2021. hal-03404664

\author{
HAL Id: hal-03404664 \\ https://hal.science/hal-03404664
}

Submitted on 26 Oct 2021

HAL is a multi-disciplinary open access archive for the deposit and dissemination of scientific research documents, whether they are published or not. The documents may come from teaching and research institutions in France or abroad, or from public or private research centers.
L'archive ouverte pluridisciplinaire HAL, est destinée au dépôt et à la diffusion de documents scientifiques de niveau recherche, publiés ou non, émanant des établissements d'enseignement et de recherche français ou étrangers, des laboratoires publics ou privés. 


\title{
Effects of particles size on the overall strength of nanocomposites: Molecular Dynamics simulations and theoretical modeling
}

\author{
Antoine Lucchetta ${ }^{1}$, Stella Brach ${ }^{2}$, Djimédo Kondo $^{1}$ \\ ${ }^{1}$ Sorbonne Université, Institut Jean Le Rond d'Alembert, UMR 7190 CNRS, 4, Place Jussieu, 75252 Paris Cedex 05, France \\ ${ }^{2}$ LMS, CNRS, Ecole Polytechnique, Institute Polytechnique de Paris, Palaiseau, 91128, France
}

\begin{abstract}
We perform Molecular Dynamics simulations to investigate the strength properties of particulate reinforced nanocomposites. For a fixed reinforcement volume fraction, the effective strength increases as the inclusion size decreases. We further develop a kinematic limit analysis approach, which delivers theoretical estimates of the effective strength. The model is first assessed in the absence of size effects by comparison with data from available literature. An extension to nanocomposites is then proposed, accounting for the presence of surface stresses at the matrix/inclusion interface. Numerical data are used to calibrate the interfacial strength, which is found to be a size-dependent property.
\end{abstract}

Keywords: Nanocomposites, Strength properties, Limit analysis, Molecular Dynamics computations

\section{Introduction}

Nanocomposites have inspired an intense research activity in many engineering fields, leading to the design of a number of high-performance devices. However, while there exist well-established homogenization theories for conventional heterogeneous media [18, 29], few up-scaling models can be found in literature for materials whose leading length-scale is in the order of submicrons or yet nanometers. As modern technologies increasingly rely on nanostructured materials, one of the current challenges to solid mechanics is understanding how the effective properties depend on the characteristic size of the material heterogeneity, eventually allowing for the development of novel up-scaling models. Inclusions size effects in nanocomposites have been reported experimentally (see for instance $[3,16,31,12,13]$ ). As regards theoretical modeling, extensions of the HashinShtrikman bounds [9, 10], Mori-Tanaka [15] and selfconsistent [25] schemes have been proposed to predict the effective stiffness of those materials. In these models, surface energies are believed to play an important role due to the high surface-to-volume ratio which has been numerically investigated by many authors among which one can mention [1, 23]. On the other hand, non-linear homogenization models have been proposed mainly for nanoporous media $[5,6,28]$. As such, the present contribution aims at investigating the effective strength of composites made of a plastic matrix rein- forced by nanoparticles. To this end, we use Molecular Dynamics to understand the impact of size effects on the overall constitutive behavior and kinematic limit analysis to theoretically estimate the macroscopic strength.

\section{Molecular Dynamics simulations}

The effective constitutive behavior of a nanocomposite sample is investigated numerically via Molecular Dynamics. Simulations are run in LAMMPS [30], following the work [4].

The computational domain is a cubic cell (see Fig. 1) comprised of an aluminum matrix and a spherical nickel nanoparticle. Both materials have a FCC crystallographic structure, characterized by a relatively low degree of anisotropy with respect to other lattices. A Cartesian reference system is introduced with basis $\left\{\underline{e}_{1}, \underline{e}_{2}, \underline{e}_{3}\right\}$. The crystallographic directions [100], [010] and [001] are aligned with $\underline{e}_{1}, \underline{e}_{2}$ and $\underline{e}_{3}$ respectively. The initial velocity vectors of the atoms are computed by equilibrating the sample at the constant temperature $300 \mathrm{~K}$. The numerical simulations have been conducted in the canonical ensemble (NVT) with this temperature. The effect of temperature has been assessed in preliminary studies, and mainly consisted in a reduction of the stress peak when temperature was increased. The same effect has been observed for nanoporous materials (see for instance [4]), due the in- 
creased atoms mobility. Also, no substantial difference was observed in terms of effective strength and overall behavior by letting the temperature freely evolve during the loading process. The time discretization is $\Delta T=1$ femtosecond. The reinforcement volume fraction is $f=1 \%$, while the particle radius takes the values $0.25,0.5,0.75$ and $1.25 \mathrm{~nm}$. An uniaxial test is performed by applying the macroscopic strain-rate tensor D

$$
\boldsymbol{D}=D_{11} \underline{e}_{1} \otimes \underline{e}_{1},
$$

where $D_{11}=5 e 9 \AA / p s$ is the strain rate (see $[4,20]$ for sensitivity analyses), while the effective stress tensor is

$$
\boldsymbol{\Sigma}=\Sigma_{11} \underline{e}_{1} \otimes \underline{e}_{1}+\Sigma_{33}\left(\underline{e}_{2} \otimes \underline{e}_{2}+\underline{e}_{3} \otimes \underline{e}_{3}\right)
$$

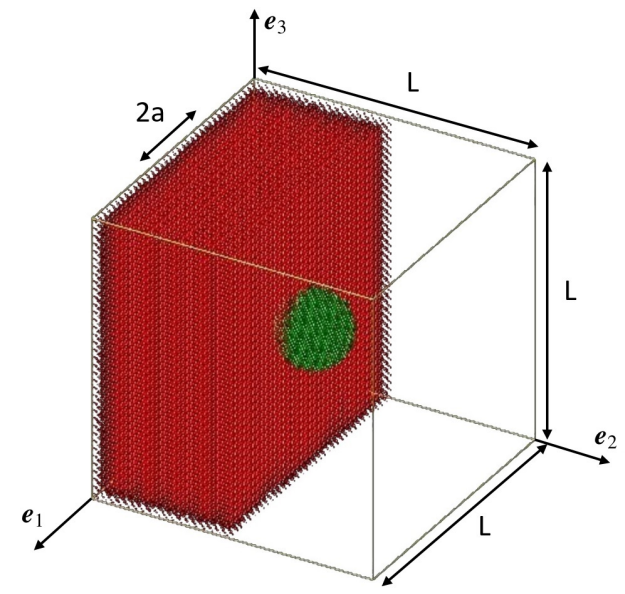

Figure 1: Molecular Dynamics simulations. Computational domain.

The stress is computed via the virial formula $[35,36]$ :

$$
\boldsymbol{\Sigma}(\bar{t})=-\frac{1}{V_{\mathrm{at}}} \sum_{i=1}^{N}\left(m_{\mathrm{i}} \underline{v}_{\mathrm{i}} \otimes \underline{v}_{\mathrm{i}}+\sum_{j \neq i, j=1}^{N} f_{\mathrm{ij}} \frac{\underline{r}_{\mathrm{ij}} \otimes \underline{r}_{\mathrm{ij}}}{r_{\mathrm{ij}}}\right)
$$

where $V_{\text {at }}$ is the total atomic volume, $m_{\mathrm{i}}$ and $\underline{v}_{\mathrm{i}}$ are the mass and velocity of atom $i, f_{\mathrm{ij}}$ is the interaction force between atoms $i$ and $j$, and $\underline{r}_{\mathrm{ij}}$ their relative distance. The virial formula (3) has been broadly used in Molecular Dynamics to obtain an average measure of the atomistic stress [40]. Equivalence to the continuum Cauchy stress has been shown in [34]. Applications to heterogeneous media can be found for instance in [37, 26, 27]. Computed stress-strain curves are shown in Fig. 2. After an initial elastic phase, the sample begins to plastically deform. The stress increases until reaching a peak

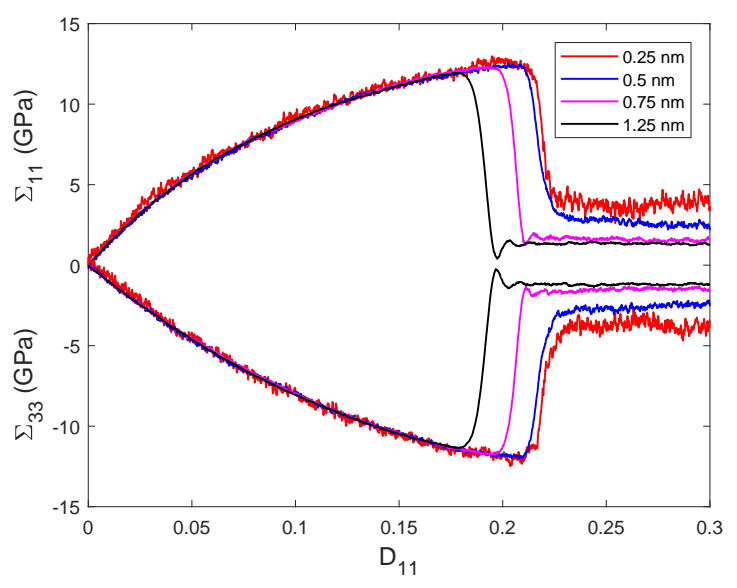

Figure 2: Effective stress components $\Sigma_{11}$ and $\Sigma_{33}$ as a function of the loading parameter for different inclusion sizes. $f=1 \%$.

value. At this point, the sample fails and the stress subsequently drops. Further oscillations are due to the release/locking of dislocation systems as well as to the interaction with the surrounding periodic cells. The effective strength is defined as the maximum bearing capacity of the material, hence the maximum value of the equivalent stress measure $\left(\Sigma_{11}-\Sigma_{33}\right) / \sigma_{0}$ in Fig. 3, with $\sigma_{0}=15.86 \mathrm{GPa}$ the matrix strength. Note that the stress peak used to defined the effective strength is due to dislocations pile-up, locking and subsequent unlocking of sessile assemblies. The small fluctuations observed in the stress-strain curve after the stress peak are mainly due to dislocation interactions through the cell boundaries. We believe that those fluctuations are not influenced by the average measure of stress, as they tend to disappear when the temperature (hence atoms mobility) is increased.

Results in Fig. 4 show that the effective strength is a size-dependent property: for a given volume fraction, it increases with decreasing the size of the inclusion reaching values 2 times higher than the strength of the matrix constituent for the smallest radius. Importantly, such a dependency cannot be predicted by conventional homogenization models, as these account for the material geometry only through relative measures such as volume fractions or aspect ratios. Conversely, our simulations show that the effective property also depends on the absolute value of the heterogeneity characteristic size. 


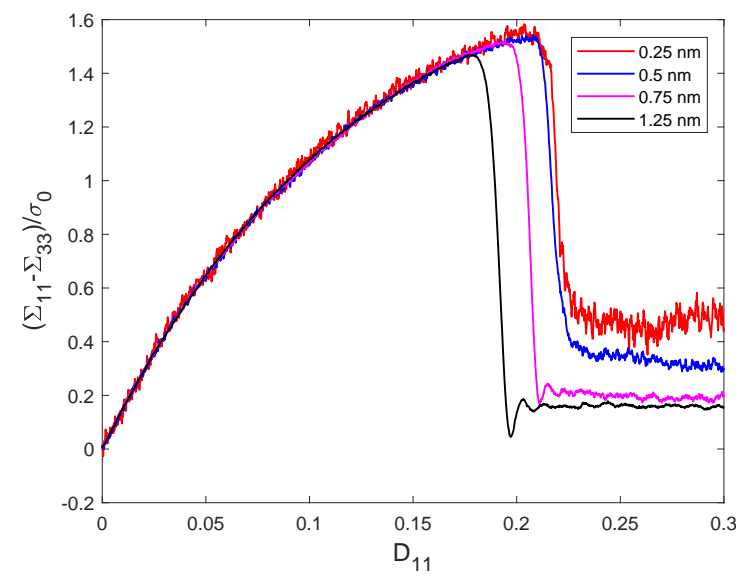

Figure 3: Equivalent stress measure $\left(\Sigma_{11}-\Sigma_{33}\right) / \sigma_{0}$ as a function of the loading parameter for different inclusion sizes. $f=1 \%$ and $\sigma_{0}=$ $15.86 \mathrm{GPa}$.

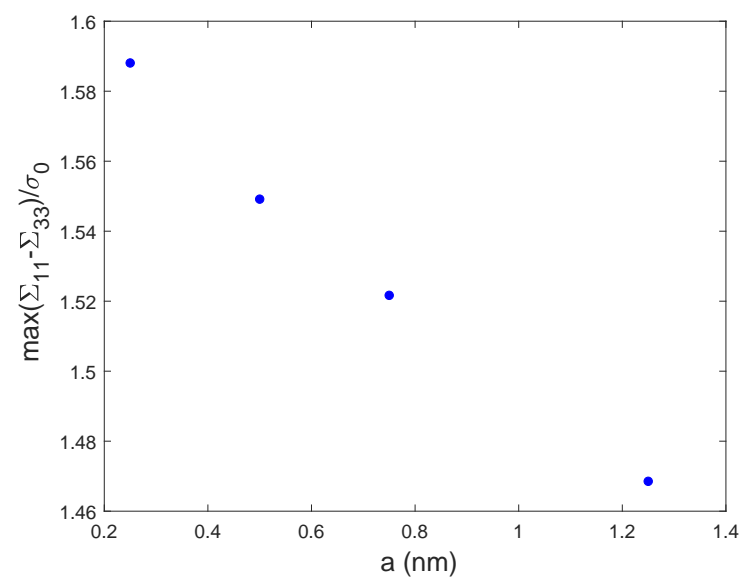

Figure 4: Computed values of effective strength $\max \left(\Sigma_{11}-\Sigma_{33}\right) / \sigma_{0}$ as a function of the inclusion size. $f=1 \%$ and $\sigma_{0}=15.86 \mathrm{GPa}$.

\section{Kinematic limit analysis}

Based on our numerical results, we propose a kinematic limit analysis approach to predict the effective strength of nanocomposites. First, we assume that the characteristic size of the inclusions is large enough for size effects to be negligible. The macroscopic strength criterion obtained via the proposed procedure is assessed by comparison with the available literature. We then consider smaller inclusion sizes, for which size effects are expected to occur. The proposed approach is extended to the case of nanocomposites, thus predicting not only the influence of the reinforcement volume frac- tion on the effective strength but also its dependence on the inclusion size.

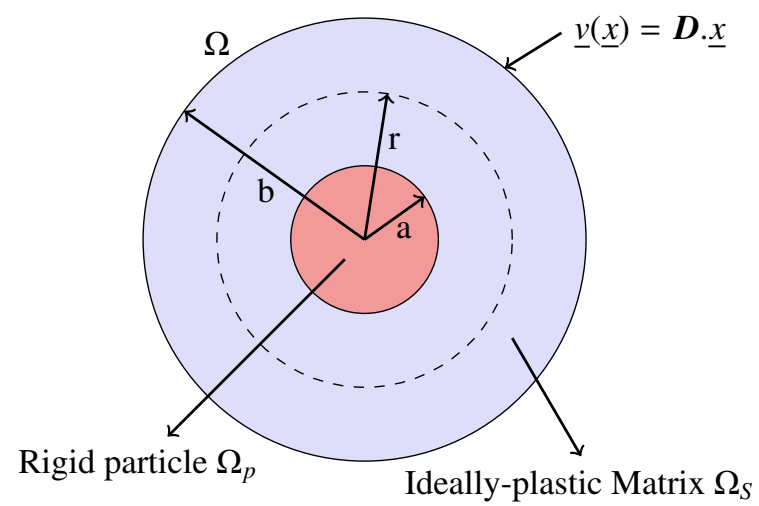

Figure 5: Theoretical model. Unit cell.

\subsection{Methods}

Consider a representative volume element whose unit cell is the composite sphere $\Omega$ shown in Fig. 5. The cell is comprised of an ideally-plastic isotropic material $\Omega_{\mathrm{s}}$ reinforced with a linear elastic spherical particle $\Omega_{\mathrm{p}}$. Both materials are assumed to be rigid. The inner and outer radii are $r=a$ and $r=b$ respectively, the reinforcement volume fraction is $f=a^{3} / b^{3}$. The outer boundary $\partial \Omega$ of the unit cell is subjected to the velocity field $\underline{v}(\underline{x})=\boldsymbol{D} \cdot \underline{x}$, where $\boldsymbol{D}$ is the macroscopic strain-rate tensor and $\underline{x}$ is a position vector. The set of kinematically-admissible velocity field is $K_{\mathrm{c}}=\{\underline{v} / \underline{v}(\underline{x})=\boldsymbol{D} \cdot \underline{x}, \forall \underline{x} \in \partial \Omega\}$, while the plastic admissibility condition reads as $\operatorname{div}(\underline{v})=\operatorname{tr}(\boldsymbol{d})=0$ where $\boldsymbol{d}=\left(\nabla(\underline{v})+\nabla(\underline{v})^{T}\right) / 2$ is the local strain-rate tensor.

The material obeys to a von Mises strength criterion

$$
f(\sigma)=\sqrt{\frac{1}{2} \sigma_{d}: \sigma_{d}}-k \leq 0 \quad \text { with } \quad k=\frac{\sigma_{0}}{\sqrt{3}},
$$

where $\sigma_{\mathrm{d}}$ is the deviatoric part of the Cauchy stress tensor, $k$ the shear strength and $\sigma_{0}$ the strength under uniaxial stress. An equivalent formulation of the strength criterion is provided by the support function on the convex set $G(\underline{x})=\{\sigma / f(\sigma) \leq 0\}[32]$

$$
\pi(\boldsymbol{d})=\sup _{\boldsymbol{\sigma} \in G}\{\boldsymbol{\sigma}: \boldsymbol{d}\}= \begin{cases}+\infty & \text { if } \operatorname{tr}(\boldsymbol{d}) \neq 0 \\ k \sqrt{2 \boldsymbol{d}: \boldsymbol{d}} & \text { if } \operatorname{tr}(\boldsymbol{d})=0\end{cases}
$$

Owing to the possible discontinuity of the trial velocity across the matrix/inclusion interface $\mathcal{S}(a)$, the following 
contribution to the support function, at $r=a$, must be also considered

$$
\pi(\underline{n}, \underline{v})=\sup _{\sigma \in G}\{(\sigma \cdot \underline{n}) \cdot \underline{v}\}= \begin{cases}+\infty & \text { if } \underline{v} \cdot \underline{n} \neq 0 \\ k\|\underline{v}\| & \text { if } \underline{v} \cdot \underline{n}=0\end{cases}
$$

where $\underline{n}$ is the outer normal vector to $\mathcal{S}(a)$ and $\|$.$\| is the$ Euclidean norm.

Similar considerations allow us to identify the macroscopic admissible stress states

$$
\Sigma=\frac{\partial \Pi^{\mathrm{hom}}(\boldsymbol{D})}{\partial \boldsymbol{D}}
$$

where

$$
\Pi^{h o m}(\boldsymbol{D})=\frac{1}{|\Omega|}\left(\int_{\Omega} \pi(\boldsymbol{d}) d \Omega+\int_{\mathcal{S}(a)} \pi(\underline{n}, \underline{v}) d S\right)
$$

is the macroscopic support function.

\subsubsection{Choice of a velocity field}

Both material constituents are rigid and the matrix is assumed to be plastically incompressible. We therefore expect the composite to behave as an incompressible material at the macroscopic length-scale. Hence, we focus on the situation where the unit cell is subjected to purely deviatoric boundary conditions, that is $\boldsymbol{D}=\left(\underline{e}_{1} \otimes \underline{e}_{1}+\underline{e}_{2} \otimes \underline{e}_{2}\right) / 2-\underline{e}_{3} \otimes \underline{e}_{3}$.

A variety of velocity fields could be proposed to satisfy the plastic admissibility requirement. Among others, we refer to the expressions provided in [11] and commonly used to describe the kinematics of conventional composites [38, 39] (see [7] for nanoporous media). In a spherical reference frame, the components of the plastically-incompressible velocity field are

$$
\begin{aligned}
& v_{\mathrm{r}}=-\frac{1}{r^{2} \sin \theta} \frac{\partial}{\partial \theta}(\zeta(r, \theta) \sin \theta) \\
& v_{\theta}=\frac{1}{r} \frac{\partial}{\partial r}(\zeta(r, \theta)), \quad v_{\varphi}=0 .
\end{aligned}
$$

Under the applied loading, $\zeta(r, \theta)$ reads as

$$
\zeta(r, \theta)=\left(\frac{r^{3}}{4}+\chi(r)\right) \sin 2 \theta,
$$

where $\chi(r)$ is a polynomial function. The following ex- pressions are then obtained

$$
\begin{aligned}
& v_{\mathrm{r}}=-\frac{(1+3 \cos (2 \theta))}{r^{2}}\left(\frac{r^{3}}{4}+\chi(r)\right) \\
& v_{\theta}=\frac{\sin (2 \theta)}{r}\left(\frac{3 r^{2}}{4}+\chi^{(1)}(r)\right), \quad v_{\varphi}=0,
\end{aligned}
$$

where $\chi^{(n)}$ is the $\mathrm{n}$-th derivative of $\chi$ with respect to the radial coordinate $r$. By kinematic admissibility, function $\chi$ has to verify the conditions

$$
\chi(b)=\chi^{(1)}(b)=0,
$$

whereas plastic admissibility at the matrix/inclusion interface (see Eqs. (5) and (6)) leads to

$$
v_{r}\left(\underline{x}=a \underline{e}_{r}\right)=0 \quad \forall \theta \in[0,2 \pi],
$$

that is

$$
\chi(a)=-\frac{a^{3}}{4} .
$$

Note that condition (13) is less restrictive with respect to what originally reported in $[38,39]$.

We also note that this development is an extension of proposed work of Brach et al. [7] who developped, thanks to a limit analysis approach, an effective strength of nanoporous materials.

\subsection{Particulate reinforced composites}

The velocity field in Eqs.(9)-(14) implies $\int_{\mathcal{S}(a)} k\left|v_{\theta}(a)\right| d S=0$. The macroscopic support function (8) then reads

$$
\Pi^{h o m}(\boldsymbol{D})=\frac{1}{|\Omega|} \int_{\Omega} k \sqrt{2 \boldsymbol{d}: \boldsymbol{d}} d \Omega .
$$

This calculation can not be performed analytically. As the kinematic limit analysis method provides upperbound estimates of the macroscopic effective strength, we rely on the Cauchy-Schwarz inequality to analytically compute an upper-bound of (15). This results in

$$
\Pi^{h o m}(\boldsymbol{D})=\frac{k}{|\Omega|} \sqrt{\frac{4 \pi\left(b^{3}-a^{3}\right)}{3}} \sqrt{\mathcal{I}(\chi)},
$$

with

$$
\begin{aligned}
\mathcal{I}(\chi) & =\int_{a}^{b} \int_{\mathcal{S}(r)} 2 \boldsymbol{d}: \boldsymbol{d} d S d r \\
& =\int_{a}^{b} \mathcal{L}\left(r, \chi, \chi^{(1)}, \chi^{(2)}\right) d r .
\end{aligned}
$$

A detailed expression of $\mathcal{I}$ is reported in Appendix A. We aim at obtaining the lowest upper-bound of the 
macroscopic support function (16) such that $\chi$ complies with Eqs. (12) and (14). The stationary condition on the functional $\mathcal{L}$ for any function $\chi$ is

$$
\frac{\partial \mathcal{L}}{\partial \chi}-\frac{d}{d r}\left(\frac{\partial \mathcal{L}}{\partial \chi^{(1)}}\right)+\frac{d^{2}}{d r^{2}}\left(\frac{\partial \mathcal{L}}{\partial \chi^{(2)}}\right)=0
$$

yielding the differential equation

$$
24 \chi^{(1)}(r)-12 r \chi^{(2)}(r)+r^{3} \chi^{(4)}(r)=0,
$$

whose solution is

$$
\chi(r)=C_{1}+C_{2} \frac{b^{2}}{r^{2}}+C_{3} \frac{r^{3}}{b^{3}}+C_{4} \frac{r^{5}}{b^{5}} .
$$

Constants $C_{1}$ and $C_{2}$ can be expressed in terms of $C_{3}$ and $C_{4}$ by combining Eqs. (12) and (20). The latter are computed by imposing the admissibility condition (14), the resulting expressions only depend on the inclusion radius $a$, on the volume fraction $f$ and on $\chi^{(1)}(a)$ which is an unknow of the problem. Now we have the expression of these four constants, the functional $I(\chi)$ can be expressed in term of $\chi^{(1)}(a)$. Finally, $\chi^{(1)}(a)$ has been determined as the value which minimizes $\Pi^{\text {hom }}(\boldsymbol{D})$ expressed in Eq. (16) (see Appendix A for details).

For the considered boundary condition, Eq. (7) allows to compute the effective deviatoric strength as

$2 \Sigma_{d} D_{e q}=\Pi^{h o m}(\boldsymbol{D}), \quad\left(\right.$ with $\left.D_{e q}=\sqrt{\frac{2}{3} \boldsymbol{D}: \boldsymbol{D}}=\frac{\sqrt{3}}{2}\right)$,

that is

$$
\Sigma_{\mathrm{d}}=k \sqrt{1-f} \sqrt{\frac{2-3 f+5 f^{7 / 3}+3 f^{10 / 3}}{2-5 f+5 f^{7 / 3}-2 f^{10 / 3}}},
$$

which only depends on the shear strength $k$ of the matrix constituent, as well as on the reinforcement volume fraction $f$. Fig. 6 shows the variation of $\Sigma_{\mathrm{d}} / \sigma_{0}$ versus the volume fraction $f$, under uniaxial boundary conditions. The effective strength is equal to that of the matrix constituent for $f=0$, whereas it increases as the reinforcement volume fraction increases. For $f \rightarrow 1$, the effective strength is unbounded as it is the case for the rigid inclusion. Predictions from Eq. (22) are compared to Zhu and Zbib model [38], Garajeu and Suquet model [17] $\left(\boldsymbol{\Sigma}_{d}=\sigma_{0} \sqrt{1+f}\right)$ and numerical estimates [2]. With respect to the previous models, the proposed approach allows to effectively capture the numerical trend. We emphasize that we use the same velocity field as in Zhu and Zbib [38]. Nevertheless, contrary to these autors, the macroscopic support function $\Pi^{\text {hom }}$ has been optained after an optimization procedure. The latter leads to a better prediction of the effective strength. It is also observed that the Garajeu and Suquet model has a better prediction for small volume fraction of inclusions for which their model had been developped.

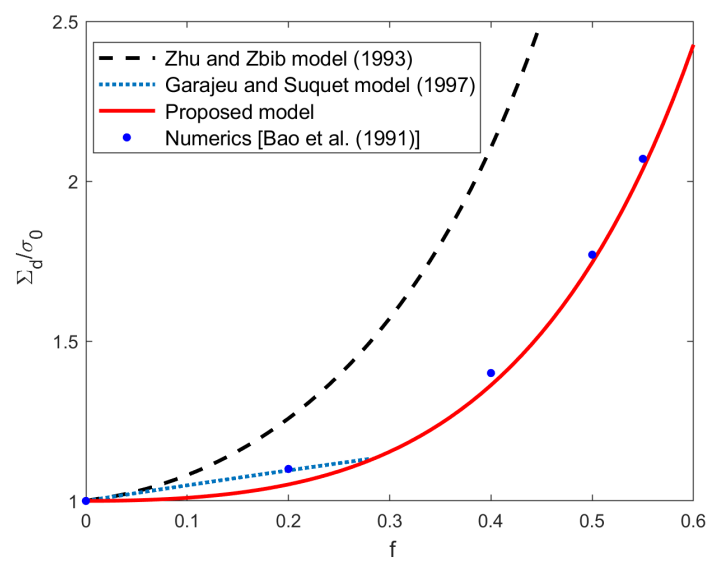

Figure 6: Normalized effective strength $\Sigma_{\mathrm{d}} / \sigma_{0}$ as a function of the reinforcement volume fraction. Comparison with the proposed model (22) (red line), Zhu and Zbib model (black line) [38], Garajeu and Suquet model (blue line) [17] and numerical estimates (blue dots) [2].

\subsection{Particulate reinforced nanocomposites}

Due to the small length-scale involved, surface stresses are expected to arise at the matrix/inclusion interface [19, 22, 33]. Following [14] and later works $[6,5,8]$, we account for the presence of such stress distributions by describing $\mathcal{S}(a)$ as a coherent imperfect interface obeying the Young-Laplace equilibrium equations

$$
\begin{aligned}
& \underline{n} \cdot[\boldsymbol{\sigma}] \cdot \underline{n}=-\operatorname{div}(\boldsymbol{\tau}): \boldsymbol{\kappa} \\
& \boldsymbol{P} \cdot[\boldsymbol{\sigma}] \cdot \underline{n}=-\nabla_{\mathcal{S}} \cdot \boldsymbol{\tau},
\end{aligned}
$$

where $\boldsymbol{P}=\mathbf{1}-\underline{n} \otimes \underline{n}, \boldsymbol{\kappa}$ is the curvature tensor and $\boldsymbol{\tau}$ is the surface stress field inducing the stress discontinuity $[\sigma]$. We assume that the interface obeys a von Mises criterion

$$
f^{\mathcal{S}}(\tau)=\sqrt{\frac{1}{2} \tau_{\mathrm{d}}: \tau_{\mathrm{d}}}-k_{\mathcal{S}} \leq 0,
$$

where $\tau_{\mathrm{d}}$ is the interface stress deviator and $k_{\mathcal{S}}=$ $\sigma_{0}^{\mathcal{S}} / \sqrt{3}$ is the interface shear strength, $\sigma_{0}^{\mathcal{S}}$ being the uniaxial tension strength expressed in $\mathrm{N} / \mathrm{m}$.

The macroscopic support function is defined as the sum of the plastic dissipation coming from the matrix constituent $\left(\Pi_{\mathrm{m}}^{\mathrm{hom}}\right)$ and the dissipation performed 
against the surface stresses at the interface $\left(\Pi_{\mathcal{S}}^{\text {hom }}\right)$

$$
\Pi^{\mathrm{hom}}(\boldsymbol{D})=\Pi_{\mathrm{m}}^{\mathrm{hom}}(\boldsymbol{D})+\Pi_{\mathcal{S}}^{\mathrm{hom}}(\boldsymbol{D}) .
$$

The first term reads as in Eqs. (15) and (16), while the interfacial contribution is (see $[14,24])$

$$
\Pi_{\mathcal{S}}^{\mathrm{hom}}(\boldsymbol{D})=\frac{1}{|\Omega|} \int_{\mathcal{S}(a)} \pi_{\mathcal{S}}\left(\boldsymbol{d}^{\mathcal{S}}\right) d S,
$$

where $\boldsymbol{d}^{\mathcal{S}}$ is the projection of the strain rate $\boldsymbol{d}$ on the plane tangent to $\mathcal{S}(a)$, while

$$
\begin{aligned}
\pi_{\mathcal{S}}\left(\boldsymbol{d}^{\mathcal{S}}\right) & =\sup _{\boldsymbol{\tau} \in G^{\mathcal{S}}}\left\{\boldsymbol{\tau}: \boldsymbol{d}^{\mathcal{S}}\right\} \\
& =2 k_{\mathcal{S}} \sqrt{\frac{1}{3}\left(d_{\theta \theta}^{2}+d_{\varphi \varphi}^{2}+d_{\theta \varphi}^{2}+d_{\theta \theta} d_{\varphi \varphi}\right)},
\end{aligned}
$$

is the support function of the interface strength domain $G^{\mathcal{S}}=\left\{\boldsymbol{\tau} / f^{\mathcal{S}}(\boldsymbol{\tau}) \leq 0\right\}$.

As in the absence of size effects, we apply the Cauchy-Schwarz inequality to analytically compute an upper-bound of Eq. (26). The resulting expression, developped in Appendix B, depends not only on the reinforcement volume fraction $f$ but also on the inclusion size $a$ and on the interface strength $k_{\mathcal{S}}$. Note that the derivative $\chi^{(1)}(a)$ has to be equal to that computed in the absence of the interface in order for the model to recover Eq. (16) when surface stresses are negligible. Finally, the effective deviatoric strength is

$$
\Sigma_{\mathrm{d}}=\Sigma_{\mathrm{d}}^{*}+\frac{k_{\mathcal{S}}}{a} \Sigma_{\mathrm{d}}^{* *}
$$

where $\Sigma_{\mathrm{d}}^{*}$ reads as in Eq. (22) whereas the expression of $\Sigma_{\mathrm{d}}^{* *}$ is detailed in Appendix B as a function of the volume fraction $f$. As the size of the inclusion becomes larger, surface stresses fade (i.e., $k_{\mathcal{S}}=0$ ) and the effective strength of the nanocomposite tends to that computed for a conventional particulate reinforced material. On the other hand, for a given volume fraction $f, \Sigma_{\mathrm{d}}$ increases with reducing the inclusion size.

In Eq.(28), we do not have any information on the interface shear strength $k_{S}$. To estimate it, we perform an inverse identification to get variation of $k_{S}$ as a function of the inclusion size $a$. The result of the numerical calibration, presented in the form of $\ln \left(k_{S}\right)$ with respect to $\ln (a)$ is shown in Fig.7. This leads to a power law

$$
k_{\mathcal{S}}=676.55 \times a^{0.9370}, \quad(a \text { expressed in } \mathrm{nm}),
$$

represented in Fig. 8. Finally, inserting (29) in (28) we

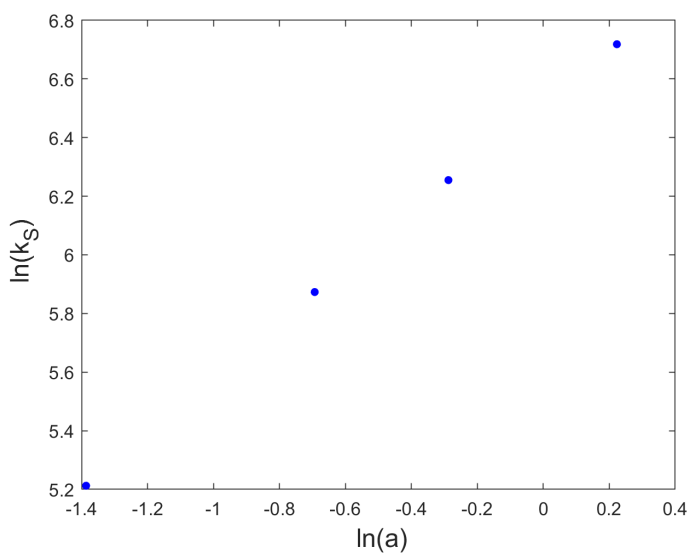

Figure 7: Variation of $\ln \left(k_{s}\right)$ with respect to $\ln (a), a$ in nanometer.

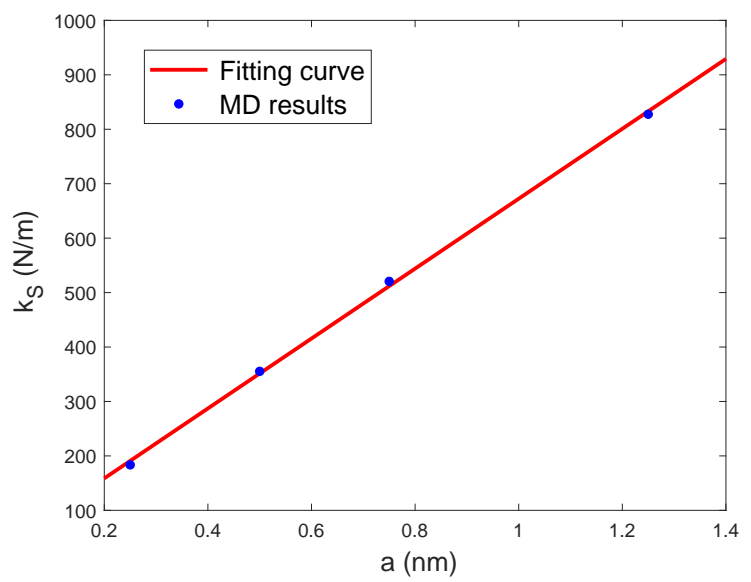

Figure 8: Interfacial strength $k_{\mathcal{S}}$ as a function of the inclusion size $a$. After calibration with the numerical data, a power law of the form (29) has been obtained. Variation of the fitting expression of $k_{S}$ (red line) and the numerical data of $k_{S}$ obtained numerically (blue dots).

get a good approximation of the Molecular Dynamics results (see Fig.9).

\section{Summary and perspectives}

This paper investigates the effective strength of particulate composites reinforced with spherical nanoinclusions. Our approach is twofold. First, we perform Molecular Dynamics simulations to understand the influence of the inclusion size on the overall constitutive behavior. We found that the effective strength increases as the inclusion size reduces, although the reinforcement volume fraction is kept constant. Sec- 


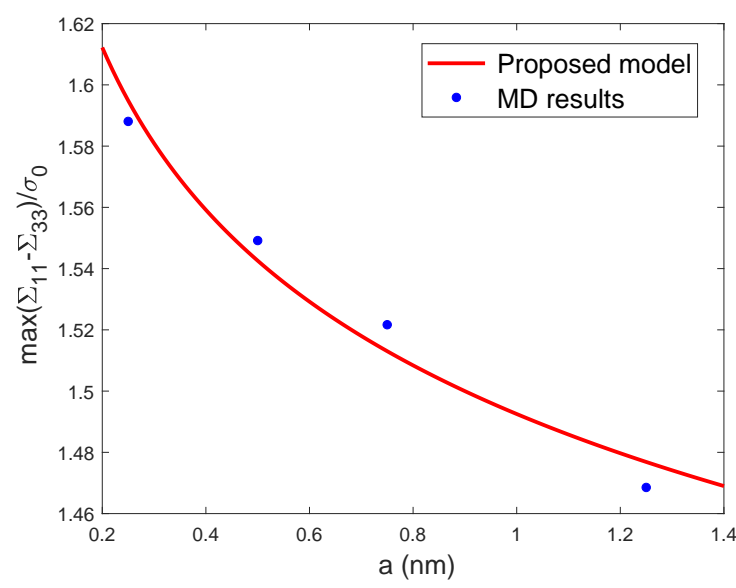

Figure 9: Comparison between Molecular Dynamics results (blue dots) and the theoretical prediction in Eq. (28) (red line). The interfacial strength $k_{\mathcal{S}}$ has been calibrated into numerical data as shown in Fig. 8.

ond, we develop a kinematic limit analysis approach to theoretically predict the effective strength of such materials. We start by neglecting size effects, the resulting model being assessed by comparison with data from previous models and simulations. Then, we extend the proposed formulation to nanocomposites by accounting for the role played by surface stresses at the matrix/inclusion interface. A final comparison between theory and numerics leads to the important result that the interfacial strength is a size-dependent parameter.

Further investigations are certainly needed, including a comprehensive characterization of the effective strength in the setting of crystal plasticity as well as a thorough assessment of the interface modeling.

\section{References}

[1] D.P. Bach, D. Brancherie, L. Cauvin, Size effect in nanocomposites: XFEM/level set approach and interface element approach, Finite Elements in Analysis and Design, 165 (2019) 41-51.

[2] G. Bao, J. W. Hutchinson, R. M. McMeeking, Particle reinforcement of ductile matrices against plastic flow and creep, Acta metallica Material, 39 (1991) 1871-1882.

[3] A. S. Blivi, F. Benhui, J. Bai, D. Kondo, F. Bédoui, Experimental evidence of size effect in nano-reinforced polymers : Case of silica reinforced PMMA, Polymer Testing, 56 (2016) 337-343.

[4] S. Brach, L. Dormieux, D. Kondo, G. Vairo, A computational insight into void-size effects on strength properties of nanoporous materials, Mechanics of Materials, 101 (2016) 102-117.

[5] S. Brach, L. Dormieux, D. Kondo, G. Vairo, Strength properties of nanoporous materials: A 3-layered based non-linear homoge- nization approach with interface effects, International Journal of Engineering Science, 115 (2017) 28-42.

[6] S. Brach, L. Dormieux, D. Kondo, G. Vairo, Nanoporous materials with a general isotropic plastic matrix: Exact limit state under isotropic loadings, International Journal of Plasticity, 89 (2017) $1-28$.

[7] S. Brach, L. Dormieux, D. Kondo, G. Vairo, Deviatoric Strength of Nanoporous Materials: A Limit Analysis Approach, Springer Series in Solid and Structural Mechanics, 8 (2017) 153-166.

[8] S. Brach, K. Anoukou, D. Kondo, G. Vairo, Limit analysis and homogenization of nanoporous materials with a general isotropic plastic matrix, International Journal of Plasticity, 105 (2018) 2461.

[9] S. Brisard, L. Dormieux, D. Kondo, Hashin-Shtrikman bounds on the bulk modulus of a nanocomposite with spherical inclusions and interface effects, Computational Materials Science, 48 (2010) 589-596.

[10] S. Brisard, L. Dormieux, D. Kondo, Hashin-Shtrikman bounds on the shear modulus of a nanocomposite with spherical inclusions and interface effects, Computational Materials Science, 50 (2010) 403-410.

[11] B. Budiansky, J. W. Hutchinson, S. Slutsky, Void growth and collapse in viscous solids, in : Mechanics of Solids : The Rodney Hill 60th Anniversary Volume, H. G. Hopkins, M. J. Sewell, Pergamon Press, Oxford, (1982) 13-45.

[12] L. Cauvin, D. Kondo, M. Brieu, N. Bhatnagar Mechanical properties of polypropylene layered silicate nanocomposites: Characterization and micro-macro modelling, Polymer Testing, 29 (2010) 245-250.

[13] L. Cauvin, D. Kondo, M. Brieu, N. Bhatnagar Mechanical behavior of a PP platelet-reinforced nanocomposite: Experimental characterization and two scale modeling of linear and non-linear response, Materials Science and Engineering A, 527 (2010) 1102-1108.

[14] L. Dormieux, D. Kondo, An extension of Gurson model incorporating interface stresses effects, International Journal of Engineering Science, 48 (2010) 575-581.

[15] H.L. Duan, J. Wang, Z.P. Huang, B.L. Karihaloo, Sizedependent effective elastic constants of solids containing nanoinhomogeneities with interface stress, Journal of the Mechanics and Physics of Solids, 53 (2005) 1174-1196.

[16] T. D. Fornes, D. R. Paul, Modeling properties of nylon 6/caly nanocomposites using composite theories, Polymer, 44 (2003) 4993-5013.

[17] M. Garajeu, P. Suquet, Effective properties of porous ideally plastic or viscoplastic materials containing rigid particles. Journal of the Mechanics and Physics of Solids, 45 (1997) 873-902.

[18] Z. Hashin, S. Shtrikman, A variational approach to the theory of the elastic behaviour of multiphase materials, Journal of the Mechanics and Physics of Solids, 11 (1963) 127-140.

[19] S. F. Hassan, M. Gupta, Development of nano- $\mathrm{Y}_{2} \mathrm{O}_{3}$ containing magnesium nanocomposites using solidification processing, Journal of Alloys and Compounds, 429 (2007) 176-183.

[20] M. F. Horstemeyer, M. I. Baskes, S. J. Plimpton, Length scale and time scale effects on the plastic flow of fcc metals, Acta Materiala, 49 (2001) 4363-4374.

[21] D. Hull, D.J. Bacon, Introduction of Dislocations (Fifth Edition), Department of Engineering, Materials Science and Engineering, University of Liverpool, United Kingdom, (2011).

[22] S. Kamrani, R. Riedel, S. M. Seyed Reihani, Effect of Reinforcement Volume Fraction on the Mechanical Properties of Al-SiC Nanocomposites Produced by Mechanical Alloying and Consolidation, Journal of Composite Materials, 44 (2009) 313326.

[23] T.T. Le, J. Guilleminot, C. Soize, Stochastic continuum model- 
ing of random interphases from atomistic simulations. Application to a polymer nanocomposite, Computer methods in applied mechanics and engineering, 303 (2016) 430-449.

[24] H. Le Quang, Q.-C. He, Variational principles and bounds for elastic inhomogeneous materials with coherent imperfect interfaces, Mechanics of Materials, 40 (2008) 865-884.

[25] P. Li, Q. Wang, S. Shi, Differential scheme for the effective elastic properties of nano-particle composites with interface effect, Computational Materials Science, 50 (2011) 3230-3237.

[26] Li, Jia and Liu, Bin and Fang, QiHong and Huang, ZaiWang and Liu, YouWen, Atomic-scale strengthening mechanism of dislocation-obstacle interaction in silicon carbide particlereinforced copper matrix nanocomposites, Ceramics International, 43 (2017) 3839-3846.

[27] Mi, Changwen and Buttry, Daniel A and Sharma, Pradeep and Kouris, Demitris A, Atomistic insights into dislocation-based mechanisms of void growth and coalescence, Journal of the Mechanics and Physics of Solids, 59 (2011) 1858-1871.

[28] V. Monchiet, D. Kondo, Combined voids size and shape effects on the macroscopic criterion of ductile nanoporous materials, International Journal of Plasticity, 43 (2013) 20-41.

[29] T. Mori, K. Tanaka, Average stress in matrix and average elastic energy of materials with misfitting inclusions, Acta Metallurgica, 21 (1973) 571-574.

[30] S. J. Plimpton, Fast parallel algorithms for short-range molecular dynamics, Journal of Computational Physics, 117 (1995) 119.

[31] E. Reynaud, T. Jouen, C. Gauthier, G. Vigier, J. Varlet, Nanofillers in polymeric matrix : a study on silica reinforced PA6, Polymer, 42 (2001) 8759-8768.

[32] J. Salençon, Calcul à la rupture en analyse limite, Presse de l'Ecole nationale des ponts et chaussés, Paris, (1983).

[33] Khin Sandar Tun, M. Gupta, Improving mechanical properties of magnesium using nano-yttria reinforcement and microwave assisted powder metallurgy method, Composites Science and Technology, 67 (2007) 2657-2664.

[34] Subramaniyan, Arun K and Sun, CT, Continuum interpretation of virial stress in molecular simulations, International Journal of Solids and Structures, 45 (2008) 4340-4346.

[35] Swenson, Robert J, Comments on virial theorems for bounded systems, American Journal of Physics, 51 (1983) 940-942.

[36] Tsai, DH, The virial theorem and stress calculation in molecular dynamics, The Journal of Chemical Physics, 70 (1979) 13751382 .

[37] Zhao, KJ and Chen, CQ and Shen, YP and Lu, TJ, Molecular dynamics study on the nano-void growth in face-centered cubic single crystal copper, Computational Materials Science, 46 (2009) 749-754.

[38] H. T. Zhu, H. M. Zbib, A continuum model for flow strength of metal-matrix composites, Scripta Metallurgica et Materiala, 28 (1993) 1323-1328

[39] H. T. Zhu, H. M. Zbib, A macroscopic model for plastic flow in metal-matrix composite, International Journal of Plasticity, 11 (1995) 471-499.

[40] Zimmerman, Jonathan A and WebbIII, Edmund B and Hoyt, JJ and Jones, Reese E and Klein, PA and Bammann, Douglas J, Calculation of stress in atomistic simulation, Modelling and simulation in materials science and engineering, 12 (2004) S319. 


\section{Appendix A. Expression of $\chi^{(1)}(a)$}

The function $\mathcal{I}(\chi)$ defined in Eq. (17) reads

$$
\begin{aligned}
\mathcal{I}(\chi) & =\int_{a}^{b} \int_{\mathcal{S}(r)} 2 \boldsymbol{d}: \boldsymbol{d} d S d r \\
& =\frac{4}{15} \int_{a}^{b}\left[-768 r \chi(r) \chi^{(1)}(r)-32 r^{3} \chi^{(1)}(r) \chi^{(2)}(r)+24 r^{5} \chi^{(2)}(r)+208 r^{2}\left(\chi^{(1)}(r)\right)^{2}+72 r^{4} \chi^{(1)}(r)+\right. \\
& \left.96 r^{2} \chi(r) \chi^{(2)}(r)+864(\chi(r))^{2}+8 r^{4}\left(\chi^{(2)}(r)\right)^{2}+45 r^{6}\right] \frac{d r}{r^{4}},
\end{aligned}
$$

with $\chi$ expressed in Eq. (20). The constants $C_{1}$ and $C_{2}$ can be expressed by combining Eqs. (12) and (20) and read as

$$
C_{1}=-\frac{1}{2}\left(5 C_{3}+7 C_{4}\right), \quad C_{2}=\frac{1}{2}\left(3 C_{3}+5 C_{4}\right)
$$

From the admissibility condition (14), constants $C_{3}$ and $C_{4}$ read as

$$
\begin{gathered}
C_{3}=-\frac{5 a^{3}\left(f^{7 / 3}-1\right)+2 a \chi^{(1)}(a)\left(2 f^{7 / 3}-7 f^{2 / 3}+5\right)}{2\left(4 f^{10 / 3}-25 f^{7 / 3}+42 f^{5 / 3}-25 f+4\right)}, \\
C_{4}=\frac{3 a^{3}\left(f^{4 / 3}+f+f^{2 / 3}+f^{1 / 3}+1\right)+2 a \chi^{(1)}(a)\left(2 f^{4 / 3}+2 f+2 f^{2 / 3}-3 f^{1 / 3}-3\right)}{2\left(f^{1 / 3}-1\right)^{3}\left(4 f^{2}+16 f^{5 / 3}+40 f^{4 / 3}+55 f+40 f^{2 / 3}+16 f^{1 / 3}+4\right)},
\end{gathered}
$$

with

$$
\chi^{(1)}(a)=\frac{3 b^{2}\left(5 f^{5 / 3}+10 f^{2}+8 f^{7 / 3}+6 f^{8 / 3}+4 f^{3}+2 f^{10 / 3}\right)}{4\left(1-f^{1 / 3}\right)\left(2+6 f^{1 / 3}+12 f^{2 / 3}+15 f+15 f^{4 / 3}+12 f^{5 / 3}+6 f^{2}+2 f^{7 / 3}\right)},
$$

which is the solution of the minimization of $\Pi^{\text {hom }}(\boldsymbol{D})$ expressed in Eq. (16).

\section{Appendix B. Expression of $\Sigma_{d}^{* *}$}

The interfacial contribution is (see $[14,24]$ )

$$
\Pi_{\mathcal{S}}^{\operatorname{hom}}(\boldsymbol{D})=\frac{1}{|\Omega|} \int_{\mathcal{S}(a)} \pi_{\mathcal{S}}\left(\boldsymbol{d}^{\mathcal{S}}\right) d S
$$

where $\boldsymbol{d}^{\mathcal{S}}$ is the projection of the strain rate $\boldsymbol{d}$ on the plane tangent to $\mathcal{S}(a)$, while

$$
\begin{aligned}
\pi_{\mathcal{S}}\left(\boldsymbol{d}^{\mathcal{S}}\right) & =\sup _{\boldsymbol{\tau} \in G^{\mathcal{S}}}\left\{\boldsymbol{\tau}: \boldsymbol{d}^{\mathcal{S}}\right\} \\
& =2 k_{\mathcal{S}} \sqrt{\frac{1}{3}\left(d_{\theta \theta}^{2}+d_{\varphi \varphi}^{2}+d_{\theta \varphi}^{2}+d_{\theta \theta} d_{\varphi \varphi}\right)},
\end{aligned}
$$

is the support function of the interface strength domain $G^{\mathcal{S}}=\left\{\tau / f^{\mathcal{S}}(\tau) \leq 0\right\}$. As in the absence of size effects, we apply the Cauchy-Schwarz inequality to analytically compute an upper-bound of Eq. (B.1), one gets

$$
\Pi_{\mathcal{S}}^{\mathrm{hom}}(\boldsymbol{D}) \leq \frac{2 a k_{S}}{|\Omega|} \sqrt{\frac{4 \pi}{3}} \sqrt{\int_{\mathcal{S}(a)}\left(d_{\theta \theta}^{2}+d_{\varphi \varphi}^{2}+d_{\theta \varphi}^{2}+d_{\theta \theta} d_{\varphi \varphi}\right) d \mathcal{S}} .
$$


As we integrate the sum over the sphere of radius $r=a$, first, we have to compute the sum

$$
d_{\theta \theta}^{2}(a)+d_{\varphi \varphi}^{2}(a)+d_{\theta \varphi}^{2}(a)+d_{\theta \theta}(a) d_{\varphi \varphi}(a)=\frac{(9+8 \cos 2 \theta+7 \cos 4 \theta)\left(4 \chi^{(1)}(a)+3 a^{2}\right)^{2}}{32 a^{4}} .
$$

We then obtained the following expression for the integral over $\mathcal{S}(a)$ :

$$
\int_{\mathcal{S}(a)}\left(d_{\theta \theta}^{2}+d_{\varphi \varphi}^{2}+d_{\theta \varphi}^{2}+d_{\theta \theta} d_{\varphi \varphi}\right) d \mathcal{S}=\frac{11 \pi\left(4 \chi^{(1)}(a)+3 a^{2}\right)^{2}}{15 a^{2}}
$$

By carrying out the same approximation as that made in 3.2 for $\Pi^{\text {hom }}(\boldsymbol{D})$, we get

$$
\Pi_{\mathcal{S}}^{\mathrm{hom}}(\boldsymbol{D})=k_{S} \sqrt{\frac{11}{5}} \frac{4 \chi^{(1)}(a)+3 b^{2} f^{2 / 3}}{b^{3}}
$$

which depends on $\chi^{(1)}(a)$. Note that the derivative $\chi^{(1)}(a)$ has to be equal to that computed in the absence of the interface in order for the model to recover Eq. (16) when surface stresses are negligible. Finally, $\Pi_{\mathcal{S}}^{\text {hom }}(\boldsymbol{D})$ is

$$
\Pi_{\mathcal{S}}^{\mathrm{hom}}(\boldsymbol{D})=-k_{S} \frac{3}{b} \sqrt{\frac{11}{5}} \frac{2 f^{2 / 3}+4 f+6 f^{4 / 3}+8 f^{5 / 3}+10 f^{2}+5 f^{7 / 3}}{-2-4 f^{1 / 3}-6 f^{2 / 3}-3 f+3 f^{5 / 3}+6 f^{2}+4 f^{7 / 3}+2 f^{8 / 3}} .
$$

Reporting the expressions of $\Pi_{\mathrm{m}}^{\text {hom }}(\boldsymbol{D})\left(\right.$ Eqs.(15) and (16)) and $\Pi_{\mathcal{S}}^{\text {hom }}(\boldsymbol{D})$ (Eq.(B.7)) in Eq.(25), we deduce from Eq.(21) and expression of the effective strength $\Sigma_{d}$ as

$$
\Sigma_{\mathrm{d}}=\Sigma_{\mathrm{d}}^{*}+\frac{k_{\mathcal{S}}}{a} \Sigma_{\mathrm{d}}^{* *}
$$

with $\Sigma_{\mathrm{d}}^{*}$ expressed in Eq.(22) and $\Sigma_{\mathrm{d}}^{* *}$ equal to

$$
\Sigma_{\mathrm{d}}^{* *}=\sqrt{\frac{33}{5}} \frac{f^{1 / 3}\left(f^{2 / 3}+2 f+3 f^{4 / 3}+4 f^{5 / 3}+5 f^{2}+5 / 2 f^{7 / 3}\right)}{1+2 f^{1 / 3}+3 f^{2 / 3}+3 / 2 f-3 / 2 f^{5 / 3}-3 f^{2}-2 f^{7 / 3}-f^{8 / 3}} .
$$

